\title{
Sociodemographic and clinical characteristics of a diabetic population at a tertiary care center in Assam, India
}

\author{
Meghna Borah, Rohini Kanta Goswami ${ }^{1}$ \\ Department of Biochemistry, North Eastern Indira Gandhi Regional Institute of Health and Medical Sciences, Shillong, Meghalaya, ${ }^{1}$ Department of \\ Biochemistry, Assam Medical College and Hospital, Dibrugarh, Assam, India
}

\section{A B S T R A C T}

\begin{abstract}
Aims: To elucidate the sociodemographic and clinical characteristics of patients with type 2 diabetes mellitus (T2DM) attending a tertiary care hospital in Dibrugarh,Assam. Subjects and Methods: A total of I 32T2DM patients (74 males and 48 females) were included in the study. Patients were evaluated with detailed history, meticulous examination, and laboratory investigations and given a detailed interview questionnaire to fill out. Statistical Analysis Used: Graph Pad Prism, published by GraphPad Software, Inc., California. Results: The highest prevalence was found in the age group $4 \mathrm{I}-50$ years (28\%). A large number of the study population (35\%) was sedentary. It was observed that the body mass index was over the normal range in $42 \%$ of the study subjects. Central obesity was observed in 76 patients (58\%). Only $9 \%$ of the patients had managed to achieve good glycemic control $(<6.5 \%)$. In our study, $39 \%$ of the patients were taking the prescribed medications irregularly.A significant proportion of the study subjects had associated comorbidities such as hypertension (50\%), obesity (42\%), and dyslipidemia (37\%). Fasting blood glucose, postprandial blood glucose, and glycated hemoglobin levels were elevated in both males and females. The values were higher in males, but statistically, the difference was not significant. Conclusions: The present study revealed that poor glycemic control, irregular medication intake, obesity, dyslipidemia, sedentary lifestyles, and hypertension were prevalent in T2DM patients. Hence, the overall risk profile in patients from Assam was very poor and needs improvement. These data can support health professionals' actions to effectively maintain and provide a more comprehensive approach to management of T2DM.
\end{abstract}

Key words: Assam, clinical profile, Northeast India, sociodemographic characteristics, Type 2 diabetes mellitus

\section{INTRODUCTION}

In the year 2004, in diabetic care, volume 27, it was predicted that by the year 2030 India would have 79.4 million people living with diabetes. Nearly, 19 years ahead of the deadline, India had a startling estimate of 62.4 million diabetics and a further 77.2 million in the prediabetic stage. This information was revealed by a survey carried out by the Indian Council of Medical Research-India diabetes in the

\begin{tabular}{|l|l|}
\hline \multicolumn{2}{|c|}{ Access this article online } \\
\hline Quick Response Code: & Website: \\
\hline & www.josh.net \\
\hline & \\
\hline
\end{tabular}

year 2011..$^{[1]}$ Diabetes mellitus, a chronic metabolic, and endocrine disease has now reached the proportions of a global pandemic. According to statistics published by the WHO, the number of diabetics stood at 110 million in 1994 and 177 million in 2000. ${ }^{[2]}$ Now, it is at a staggering 347 million, with a major chunk of being contributed by India and China. ${ }^{[3]}$

\begin{abstract}
This is an open access article distributed under the terms of the Creative Commons Attribution-NonCommercial-ShareAlike 3.0 License, which allows others to remix, tweak, and build upon the work non-commercially, as long as the author is credited and the new creations are licensed under the identical terms.
\end{abstract}

For reprints contact: reprints@medknow.com

How to cite this article: Borah M, Goswami RK. Sociodemographic and clinical characteristics of a diabetic population at a tertiary care center in Assam, India. J Soc Health Diabetes 2017;5:37-42. 
Diabetes mellitus poses a tremendous burden to India, not only in terms of disease, disability, and death but also in the form of a financial burden. Diabetes in Indians seems to occur almost a decade earlier as compared to the Europeans. Type 2 diabetes mellitus (T2DM) is also more prevalent in India (96\% as compared to $85 \%$ worldwide). ${ }^{[4]}$ Genetic predisposition combined with lifestyle changes associated with urbanization and globalization contribute to the rapid rise in India. Due to these sheer numbers, the economic burden due to this disease is among the highest in the world. Rapid urbanization and industrialization have produced advancement on the social and economic front in developing countries such as India, which have resulted in dramatic lifestyle changes leading to lifestyle-related diseases. The transition from a traditional to modern lifestyle, consumption of diets rich in fat and calories combined with a high level of mental stress has compounded the problem further.

Understanding the sociodemographic characteristics of people with diabetes is important for many activities, including health-care planning, health education, and public health research. Before developing diabetes-related health-care products or programs, health-care planners must know the social and demographic characteristics of the target population, they hope to reach. When health educators are preparing diabetes-related educational materials or activities, or clinicians and researchers are planning treatment protocols, they need to know the demographic profile of the target audience so that appropriate programs or efforts are made available.

There is a paucity of such data from Northeast India, and this becomes yet more significant in the light of the fact that this region has significant physical, cultural, and social differences from the rest of the nation. Therefore, this present research was carried out to elucidate some sociodemographic characteristics of patients with T2DM in a tertiary care center in Dibrugarh, Assam. It is hoped that the findings of this study will contribute to the current body of knowledge by elucidating the socioeconomic status and demographic characteristics that is associated with diabetes, particularly among the adult population in Assam and help policymakers, program planners, and other agencies to create more effective interventions.

\section{SubJECTS AND METHOdS}

The present descriptive study was conducted from July 2012 to January 2013. Ethical clearance from the Institutional Ethical Committee and informed consent from patients and controls was taken. A total of 132 T2DM patients (74 males and 48 females) were included in the study. Patients were evaluated with detailed history, meticulous examination, and laboratory investigations.

Interview questionnaire which included questions that covered the subject's sociodemographic and lifestyle information was developed for use in the study. Under all aseptic and antiseptic conditions, $5 \mathrm{ml}$ of blood sample was collected from each subject from a suitable peripheral vein (preferably antecubital vein) by venipuncture using a sterile disposable syringe and divided into a sterile empty vial and an ethylenediaminetetraacetic acid (EDTA) vial. EDTA vials are used for the estimation of glycated hemoglobin. The rest of the sample was then allowed to stand for some time and then centrifuged for separation of serum. This serum was used for estimation of the other parameters such as fasting blood glucose (FBG), blood urea, and serum creatinine. Blood glucose was determined by the glucose oxidase-peroxidase method, randox reagent, and glycosylated hemoglobin by the ion-exchange resin method (Tulip group). Body mass index (BMI) was calculated by dividing weight $(\mathrm{kg})$ to height $\left(\mathrm{m}^{2}\right)$. Those with a BMI of $23.0-24.9 \mathrm{~kg} / \mathrm{m}^{2}$ were classified as overweight, $25-29.9 \mathrm{~kg} / \mathrm{m}^{2}$ as preoboese while those with a BMI $\geq 30.0 \mathrm{~kg} / \mathrm{m}^{2}$ were classified as obese and BMI $\geq 40$ was classified as pathologically obese. ${ }^{[5]}$ Central obesity was defined based on waist circumference (men $\geq 90$ and women $\geq 80 \mathrm{~cm}$ ) ${ }^{[6]}$ Blood pressure (BP) was recorded after the subjects had rested for at least $5 \mathrm{~min}$. Two readings were taken 5 min apart and mean of two was considered as the BP.

Physical activity was categorized as sedentary (light housework, sitting, standing, and driving for most of the day), moderate (an occupation or housework which involved activities that kept the subject moving for several hours a day), and heavy (heavy manual labor, a very active lifestyle, or very active sports played for several hours almost daily, any activity that involved vigorous activity lasting for several hours, almost daily).

Data were at first arranged in Microsoft Excel 2007 Worksheet, developed and published by Microsoft, Redmond, Washinghton. Data are expressed as mean \pm standard deviation for continuously distributed variables and in absolute numbers and percentages for the discrete variables. Tests of significance were done with unpaired Student's t-test as needed. Significance with $P<0.05$ was considered statistically significant.

\section{RESULTS AND OBSERVATIONS}

In the present study carried out in diagnosed cases of T2DM, the youngest case study was found to be 30 years 
of age, and the oldest to be 78 years. The age of onset of diabetes in $78 \%$ of patients was between 40 and 59 years. $76 \%$ patients were on oral hypoglycemic agents and $21 \%$ on insulin therapy and remaining (2\%) were on diet control. The duration of diabetes was between $5-10$ years in $35 \%$ and $10-15$ years in $37 \%$, more than 15 years were $20 \% .8 \%$ of patients were diagnosed with diabetes mellitus in the last 5 years. Despite being diagnosed with T2DM, 39\% of the patients were taking the prescribed medications irregularly.

\section{Sociodemographic characteristics}

The sociodemographic characteristics of the study population are shown in Table 1 . It was observed that $56 \%$ of the cases are male and $44 \%$ female with a male, female ratio of 1.4:1. The highest prevalence was found in the age group $41-50$ years (28\%) followed by the age group of $51-60$ (24\%). Most of the study population (83\%) were Hindus. $93 \%$ of the subjects was literate, with around a quarter $(25 \%)$ completing at least a primary level of education. About $22 \%$ of the subjects were unemployed, and a majority of this (19\%) were women engaged as housewives. Most of the study population (73\%) were from an urban area.

\section{Presenting symptoms}

The majority of patients (81\%) had a common complaint or symptom at presentation: tiredness or lethargy. However, it must be noted that most patients had more than one complaint at presentation. The classic symptoms of T2DM were present in a large number of patients with polyuria (49\%), polyphagia (53\%), polydipsia (49\%), and nocturia (49\%). Most of the patients $(90 \%)$ were diagnosed when they had approached a medical practitioner with a symptom or complication of T2DM. Some (8\%) were also diagnosed during a routine checkup. Half of the study population (50\%) was hypertensive, in addition to being diabetic [Table 2].

\section{Risk factors}

In the diabetic patients included in the current study, it was found that $35 \%$ was sedentary. It was also observed that the BMI was over the normal range in $42 \%$ of the study subjects. Central obesity was observed in 76 patients (58\%) with T2DM. A positive family history was found only in $17 \%$ of the patients. A large fraction of the population indulged in tobacco consumption (46\%), mainly in the form of tobacco chewing (78\%). 39\% of the diabetic population under study also consumed alcoholic beverages, the majority of which were social drinkers (81\%) [Table 3].

\begin{tabular}{lc}
\hline \multicolumn{2}{l}{ Table 1: Sociodemographic characteristics of subjects with } \\
Type 2 diabetes mellitus \\
\hline Characteristics & $\boldsymbol{n}(\%)$ \\
\hline Age (years) & \\
$<40$ & $26(20)$ \\
$41-50$ & $37(28)$ \\
$51-60$ & $32(24)$ \\
$61-70$ & $16(12)$ \\
$>70$ & $21(16)$ \\
Gender & \\
Male & $74(56)$ \\
Female & $48(44)$ \\
Marital status & \\
Unmarried & $3(2)$ \\
Married & $121(92)$ \\
Widowed/divorced & $8(6)$ \\
Religion & \\
Hindu & $83(63)$ \\
Muslim & $36(27)$ \\
Others & $13(10)$ \\
Educational status & \\
Nil & $9(7)$ \\
Primary school & $33(25)$ \\
Secondary school & $45(34)$ \\
College & $32(24)$ \\
Professional & $13(10)$ \\
Occupation & \\
Unemployed & \\
Unskilled & $29(22)$ \\
Skilled & $37(28)$ \\
Semi-professional & $31(23)$ \\
Professional & $21(16)$ \\
Retired/disabled & $10(8)$ \\
Residential area & $4(3)$ \\
Urban & \\
Rural & $97(73)$ \\
\hline & $35(27)$ \\
\hline
\end{tabular}

\begin{tabular}{lc}
\hline \multicolumn{2}{l}{ Table 2: Presenting symptoms, mode of diagnosis, and } \\
\multicolumn{3}{l}{ comorbidities of participants with Type 2 diabetes mellitus* } \\
\hline Characteristic & $\boldsymbol{n}(\%)$ \\
\hline Presenting symptoms* & \\
Polyuria & $51(39)$ \\
Nocturia & $49(37)$ \\
Polydipsia & $49(37)$ \\
Polyphagia & $53(40)$ \\
Increased tiredness/lethargy & $81(61)$ \\
Delay in healing of wounds & $4(3)$ \\
Visual impairment & $4(3)$ \\
Tingling and numbness & $22(17)$ \\
Weight loss & $14(11)$ \\
Other symptoms & $11(8)$ \\
No symptoms & $11(8)$ \\
Comorbidities & \\
Hypertension & $66(50)$ \\
Obesity & $56(42)$ \\
Dyslipidemia & $49(37)$ \\
Others & $19(14)$ \\
Mode of diagnosis & \\
Routine check up & $11(8)$ \\
Symptom/complication & $119(90)$ \\
During preoperative check up & $2(2)$ \\
\hline
\end{tabular}

*There was considerable overlap of symptoms, with most subjects presenting with more than one symptom at presentation 


\begin{tabular}{lc}
\hline \multicolumn{2}{l}{ Table 3: Distribution of nonmodifiable and modifiable risk } \\
factors in participants with Type 2 diabetes mellitus \\
\hline Risk factor & $\boldsymbol{n}(\%)$ \\
\hline Physical activity & \\
$\quad$ Sedentary & $46(35)$ \\
Moderate & $53(40)$ \\
Heavy & $33(25)$ \\
BMl grade & \\
$\quad$ Normal & $76(58)$ \\
Overweight & $51(39)$ \\
Preobese & $3(2)$ \\
Obese & $2(1)$ \\
Central obesity & \\
Present & $76(58)$ \\
Tobacco intake & \\
Yes & $61(46)$ \\
Years of intake & $12.46 \pm 8.32$ \\
Alcohol intake & \\
$\quad$ Yes & $52(39)$ \\
Years of intake & $9.42 \pm 7.68$ \\
Family history & \\
Present & $23(17)$ \\
Absent & $109(83)$ \\
\hline BMl = Body mass index
\end{tabular}

\section{Clinical characteristics}

There was found to be a significant difference in male and female subjects with respect to BMI (25.589 \pm 2.11 in males vs. $26.57 \pm 3.14$ in females), with females having a higher BMI. FBG levels, post prandial blood glucose levels and glycated hemoglobin tended to be higher in males as compared to females, but statistically, the difference was not significant. Glycemic control was poor in majority of the patients with T2DM, with $74 \%$ having glycated hemoglobin more than $7.5 \%$. It was also observed in the present study that BMI showed a positive correlation with $\mathrm{HbA1c} \%$ [Figure 1]. The Pearson correlation coefficient " $r$ " found to be 0.22 established a slight positive correlation between the two parameters [Table 4].

\section{DISCUSSION}

In the present study carried out in diagnosed cases of T2DM, the highest prevalence was found in the age group 41-50 years (28\%) followed by the age group of 51-60 (24\%). Similar findings were also observed Scott and Fisher, ${ }^{[7]}$ McNair et al., ${ }^{[8]}$ and Yoon and Kim in 2008. ${ }^{[9]}$ Age is one of the important risk factors for many noncommunicable diseases including diabetes mellitus. The main factors are that aging induces decreased insulin sensitivity and insufficient compensation of beta cell function in the face of increased insulin resistance. ${ }^{[10]}$ In the present study, it was also seen that there was a slight male preponderance in cases of diabetes mellitus with a male:female ratio of 1.4:1. The WHO Expert Committee on diabetes mellitus in the second report in

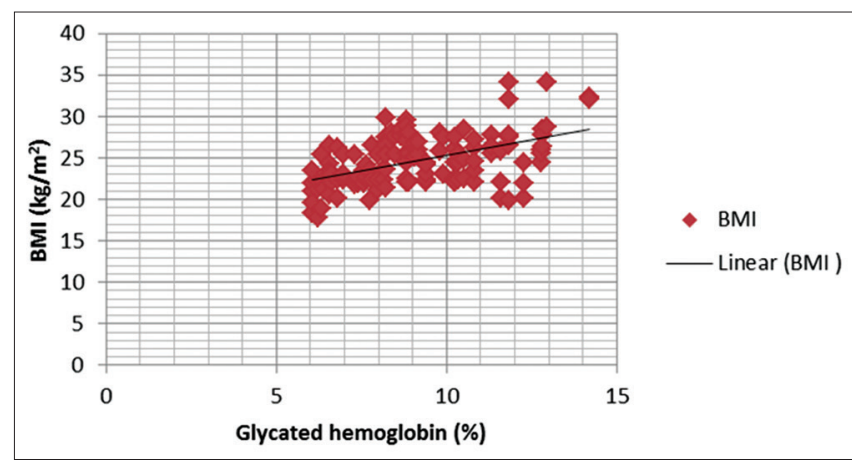

Figure 1: Correlation of glycated hemoglobin with body mass index $\left(\mathrm{kg} / \mathrm{m}^{2}\right)$ in diabetic cases

1980 mentioned that there is a slight male preponderance in Southeast Asian races. Caixas et al.$^{[11]}$ in their study of 60 patients had observed a male to female ratio of 1.14:1.

In the present study, it was observed that $7 \%$ of the study subjects were illiterate and a quarter $(25 \%)$ were educated only up to primary school level. Rubin et al. in their study observed that education appears to have a major effect on diabetes prognosis. Whether this was related to greater understanding of the illness and therefore greater commitment to self-care and therefore better access to medical care, or both, was difficult to say. ${ }^{[12]}$ In terms of marital status, it was observed that most participants are married. Psychosocial variables affect glucose fluctuations since patients realize their disease as a factor that interferes in family dynamics. An unfavorable family environment can interfere in patient compliance with treatment. It should be emphasized that organized and structured families provide a more appropriate environment to support diabetic patient health care, strongly influencing their behavior toward the disease, and making them collaborate to obtain good metabolic control.

In the present study, it was also observed that a significant proportion of the study subjects had associated comorbidities such as hypertension (50\%), obesity (42\%), and dyslipidemia (37\%). Reddy and Prabhu in their study found that $33.3 \%$ of diabetic patients also had hypertension..$^{[13]}$

Clinical randomized studies have demonstrated the benefits of a systolic BP $<140 \mathrm{mmHg}$, and diastolic $\mathrm{BP}<80 \mathrm{mmHg}$ in patients with diabetes since there is a reduction in coronary diseases, cerebrovascular accidents, and nephropathies. The main goal of arterial hypertension treatment is to reduce cardiovascular risk by obtaining appropriate BP levels, controlling risk factors and target organ lesions, which appear in the long term. ${ }^{[14]}$ 


\begin{tabular}{|c|c|c|c|c|c|}
\hline \multirow[t]{2}{*}{ Parameters } & \multicolumn{2}{|c|}{ Mean $\pm S D$} & \multirow[t]{2}{*}{$P$} & \multirow[t]{2}{*}{ Minimum } & \multirow[t]{2}{*}{ Maximum } \\
\hline & Male & Female & & & \\
\hline Age (years) & $54.14 \pm 9.76$ & $56.25 \pm 10.68$ & 0.2422 & 30 & 78 \\
\hline $\mathrm{BMI}\left(\mathrm{kg} / \mathrm{m}^{2}\right)$ & $25.589 \pm 2.11$ & $26.57 \pm 3.14$ & $0.0336^{*}$ & 17.8 & 34.6 \\
\hline FBG (mg/dL) & $168.01 \pm 65.01$ & $164.32 \pm 71.16$ & 0.7583 & 78 & 298 \\
\hline PPBG (mg/dL) & $270.08 \pm 98.62$ & $256.11 \pm 95.32$ & 0.4188 & 112 & 466 \\
\hline Systolic BP & $141.2 \pm 10.1$ & $139.9 \pm 12.39$ & 0.5091 & 94 & 168 \\
\hline Diastolic BP & $92.21 \pm 8.1$ & $88.12 \pm 9.86$ & $0.0102^{*}$ & 72 & 106 \\
\hline $\mathrm{HbA} 1 \mathrm{c}(\%)$ & $9.13 \pm 3.02$ & $9.00 \pm 2.08$ & 0.77 & 6.06 & 14.2 \\
\hline \multicolumn{6}{|c|}{ Glycemic status (HbA 1c\%), $n$ (\%) } \\
\hline $6.00-7.50$ & & & $34(26)$ & & \\
\hline $7.51-9.00$ & & & $42(32)$ & & \\
\hline $9.01-10.50$ & & & $27(20)$ & & \\
\hline$\geq 10.51$ & & & $29(22)$ & & \\
\hline
\end{tabular}

A large number of the participants (35\%) led a sedentary lifestyle. In addition to elevated BMI, central obesity was also present in $58 \%$ of the study population. This complements various studies. ${ }^{[15,16]}$ Recent studies have identified "links" between obesity and T2DM involving proinflammatory cytokines (tumor necrosis factor and interleukin-6), insulin resistance, deranged fatty acid metabolism, and cellular processes such as mitochondrial dysfunction and endoplasmic reticulum stress. These interactions are complex, with the relative importance of each unclearly defined. This issue is particularly pressing given accumulating evidence that even modest weight reduction - whether through lifestyle/ behavioral interventions, obesity medications, or bariatric surgery - can improve glycemic control and reduce diabetes risk. ${ }^{[17]}$ Recommendations concerning weight loss for diabetic patients in intensive and structured follow-up programs include education and counseling about following the food plan and doing physical activities. Maintaining the attachment between the multiprofessional team, and the patient is also an important aspect for losing and maintaining weight for long periods. It should be recalled that the genetic factor, in some cases, makes the goals more difficult to reach. ${ }^{[14]}$

In the present study, it was also observed that the FBG, postprandial blood glucose, and glycated hemoglobin levels were elevated in both males and females. The values of all three parameters were higher in males as compared to females; but statistically, the difference was not significant. In our study, only $9 \%$ of the patients had managed to achieve good glycemic control $(<6.5 \%)$. This complements a study carried out in Western India, where only $7 \%$ of the study population had good glycemic control. ${ }^{[18]}$ The results are however different from other studies such as a Swedish survey, which found that 34\% of T2DM patients had good glycemic control, ${ }^{[19]}$ study by Al-Maskari and El-Sadig, which found that 38\% T2DM of patients had good glycemic control. ${ }^{[20]}$ This difference could have arisen from the fact that the study population of our study was exclusively from a tertiary care center. The patients also had a very poor understanding of the disease process and had poor adherence to medications and interventions.

In our study, 39\% of the patients were taking the prescribed medications irregularly. Similar result was seen in a study conducted by Puria et al., where $28 \%$ patients were taking treatment irregularly. Reasons for irregular treatment in $28 \%$ were mainly failure to understand the importance of adhering to the treatment, lack of family support, and expensive medicine. ${ }^{[21]}$ The most common reason given in our study in $19 \%$ of the patients was a "feeling of relief' after the medication had been taken regularly for a few days and consumption of the prescribed medication only when the patient "felt poorly", thereby perceiving a need for the medication. Some of the patients $(9 \%)$ said the medication prescribed was too expensive to be taken everyday.

\section{Limitations}

Despite our attempt to carry out the study meticulously, it did have some limitations. First, our study was carried out in a tertiary care hospital, which may not be representative of the general population. Second, our study was carried out only for 7 months, without any follow-up of the study population. A longer duration of study combined with follow-up of the study population would certainly help in shedding greater light in this scenario. Third, most patients were not able to recall family history and were ignorant of the history of diabetes in their parents.

\section{CONCLUSION AND RECOMMENDATIONS}

Our study was an attempt to highlight the scenario of T2DM in Upper Assam. This is significant as the entire Northeast region has social, cultural, and physical 
differences with the rest of India. An insight into this region would hence prove valuable in formulating preventive and treatment policies specific for this region. Our study did bring to the forefront certain facts such as unsatisfactory metabolic control, presence of comorbidities, and irregular medication intake in a large percentage of the study population. Thus, more thought should be given to the importance of multiprofessional team education in diabetic patient care, enlightening them about nature, and progression of the disease and possible complications. They may benefit from periodical health promotion and education programs in the area of diet management, self-care, and adherence to treatment. Family should be taken into account while formulating diabetic care strategies, turning them into a collaborator in diabetic user care. Clinicians involved with managing cases of diabetes should give sufficient importance and information regarding lifestyle modifications, encouraging diet control, and exercise pattern among their patients to achieve better control of diabetes. Emphasis should be given to regular evaluation of the glycemic status and periodic checkups as well as early screening for complications. Finally, developing intervention research with diabetic users that encourage changes in habits and lifestyles, and permitting to understand what factors interfere and/or facilitate the achievement of metabolic control. In view of the obtained results and the complexity of health care required in patients with T2DM, it is concluded that increased training courses in diabetes education are needed for health-care professionals as well as a greater number of education programs are needed to raise awareness in the general population.

Ethical approval: All procedures performed in the studies involving human participants were in accordance with the ethical standards of the institutional and/or national research committee and with the 1964 Helsinki Declaration and its later amendments or comparable ethical standards.

\section{Financial support and sponsorship}

Nil.

\section{Conflicts of interest}

There are no conflicts of interest.

\section{REFERENCES}

1. Anjana RM, Pradeepa R, Deepa M, Datta M, Sudha V, Unnikrishnan $\mathrm{R}$, et al. Prevalence of diabetes and prediabetes (impaired fasting glucose and/or impaired glucose tolerance) in urban and rural India: Phase I results of the Indian
Council of Medical Research-India diabetes (ICMR-INDIAB) study. Diabetologia 2011;54:3022-7.

2. McCarty D, Zimmet P. Diabetes 1994 to 2010: Global Estimates and Projections. Melbourne: International Diabetes Institute; 1994.

3. Danaei G, Finucane MM, Lu Y, Singh GM, Cowan MJ, Paciorek CJ, et al. National, regional, and global trends in fasting plasma glucose and diabetes prevalence since 1980: Systematic analysis of health examination surveys and epidemiological studies with 370 country-years and $2 \cdot 7$ million participants. Lancet 2011;378:31-40.

4. Gupta OP, Joshi MH, Dave SK. Prevalence of diabetes in India. Adv Metab Disord 1978;9:147-65.

5. WHO. Obesity: Preventing and Managing the Global Epidemic. WHO/NUT/98. Geneva, Switzerland: World Health Organization; 1998.

6. Tan CE, Ma S, Wai D, Chew SK, Tai ES. Can we apply the National Cholesterol Education Program Adult Treatment Panel definition of the metabolic syndrome to Asians? Diabetes Care 2004;27:1182-6.

7. Scott DA, Fisher AM. The insulin and the zinc content of normal and diabetic pancreas. J Clin Invest 1938;17:725-8.

8. McNair P, Christiansen C, Christensen MS, Madsbad S, Faber OK, Binder C, et al. Development of bone mineral loss in insulin-treated diabetes: A $11 / 2$ years follow-up study in sixty patients. Eur J Clin Invest 1981;11:55-9.

9. Yoon $\mathrm{KH}, \mathrm{Kim} \mathrm{HS}$. A short message service by cellular phone in type 2 diabetic patients for 12 months. Diabetes Res Clin Pract 2008;79:256-61.

10. Chang AM, Halter JB. Aging and insulin secretion. Am J Physiol Endocrinol Metab 2003;284:E7-12.

11. Caixas A, Pérez A, Qrdóñez-Llanos J, Bonet R, Rigla M, Castellví A, et al. Lack of change of lipoprotein (a) levels by the optimization of glycemic control with insulin therapy in NIDDM patients. Diabetes Care 1997;20:1459-61.

12. Rubin RJ, Altman WM, Mendelson DN. Health care expenditures for people with diabetes mellitus, 1992. J Clin Endocrinol Metab 1994;78:809A-809F.

13. Reddy SS, Prabhu GR. Prevalence and risk factors of hypertension in urban slums, Tirupati. Indian J Community Med 2005;3:85-6.

14. American Diabetes Association. Screening for type 2 diabetes. Diabetes Care 2004;3:603.

15. Mayer-Davis EJ, Costacou T. Obesity and sedentary lifestyle: Modifiable risk factors for prevention of type 2 diabetes. Curr Diab Rep 2001;1:170-6.

16. Lieberman LS. Dietary, evolutionary, and modernizing influences on the prevalence of type 2 diabetes. Annu Rev Nutr 2003;23:345-77.

17. Eckel RH, Kahn SE, Ferrannini E, Goldfine AB, Nathan DM, Schwartz MW, et al. Obesity and type 2 diabetes: What can be unified and what needs to be individualized? Diabetes Care 2011;34:1424-30

18. Patel M, Patel IM, Patel YM, Rathi SK. A hospital-based observational study of type 2 diabetic subjects from India. Indian J Clin Pract 2013;24:141-8.

19. Holmström IM, Rosenqvist U. Misunderstandings about illness and treatment among patients with type 2 diabetes. J Adv Nurs 2005;49:146-54.

20. Al-Maskari F, El-Sadig M. Prevalence of risk factors for diabetic foot complications. BMC Fam Pract 2007;8:59.

21. Puria S, Kalia M, Mangat $C$, Goel N, Abhimanyu, Swami H. Profile of diabetes mellitus in elderly. Int J Geriatr Gerontol 2008;4:14-8. 